\title{
Headgroup-Ordered Monolayers of Uncharged Glycolipids Exhibit Selective Interactions with lons
}

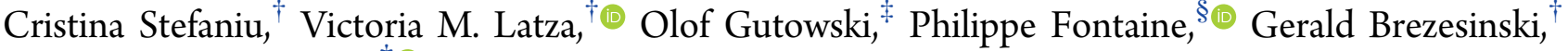 \\ and Emanuel Schneck* ${ }^{* \dagger}$ \\ ${ }^{\dagger}$ Departments of Biomaterials and Biomolecular Systems, Max Planck Institute of Colloids and Interfaces, Am Mühlenberg 1, 14476 \\ Potsdam, Germany \\ ${ }^{*}$ Deutsches Elektronen-Synchrotron (DESY), 22607 Hamburg, Germany \\ ${ }^{\S}$ Synchrotron SOLEIL, 91192 Gif-sur-Yvette Cedex, France
}

\section{Supporting Information}

ABSTRACT: Selective interactions of ions with chargeneutral saccharides can have far-reaching consequences in biological and wet-technological contexts but have so far been observed only indirectly. Here, we directly quantify by totalreflection X-ray fluorescence the preferential accumulation of ions near uncharged saccharide surfaces in the form of glycolipid Langmuir monolayers at air/water interfaces exhibiting different levels of structural ordering. Selective interactions with ions from the aqueous subphase are observed for monolayers featuring crystalline ordering of the saccharide headgroups, as determined by grazing-incidence X-ray diffraction. The attracted ion species depend on the structural motifs displayed by the ordered saccharide layer. Our results may constitute a basis to understand the salt-specific swelling of wood materials and various phenomena in membrane biophysics.

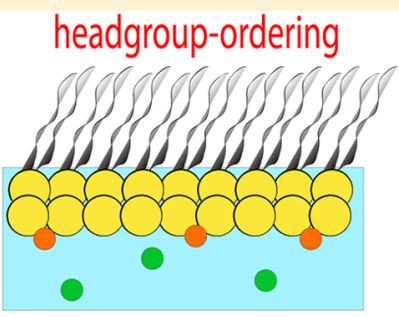

selective ion interactions
Glycolipids are essential constituents of biological membranes. Due to their structural diversity and strategic localization in various functional membrane systems, the glycolipids are considered the third alphabet of life, the sugar code. ${ }^{1}$ Glycolipids exhibit highly specific interactions with other saccharides $^{2}$ and proteins. ${ }^{3}$ Interestingly, ions are believed to promote the specific interaction of saccharide headgroups even if the latter are neither charged nor of zwitterionic character. For example, the strength of the homotypic interaction between lipid-anchored LewisX trisaccharides was reported to increase in the presence of calcium. ${ }^{4,5}$ Additional indirect evidence of selective ion interactions with saccharides is the observation of ion-specificity in the swelling of wood materials in salt solutions. ${ }^{6}$

Despite its great relevance in biology as well as for the pharmaceutical, food, cosmetic, and paper industries, ${ }^{7,8}$ our knowledge about the interaction of salts with neutral saccharides has remained limited and is based on indirect observations. In the present work, preferential interactions of ions with uncharged saccharide surfaces in the form of glycolipid Langmuir monolayers at air/water interfaces are directly quantified. Depending on the choice of the glycolipids in terms of headgroup chemistry and alkyl chain saturation, saccharide surfaces of various characteristics are realized. To this end, the highly abundant glycolipids mono- and digalactosyldiacylglycerol $^{9}$ are investigated in their chainsaturated and natural unsaturated forms (MGDG-sat, MGDGunsat, DGDG-sat, and DGDG-unsat; see Figure 1A,F). In

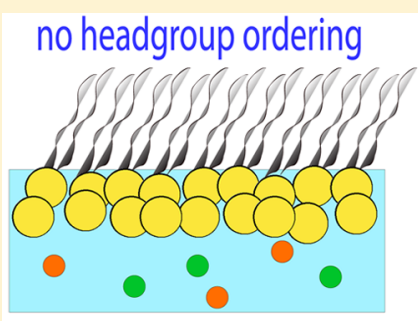

no selective ion interactions addition, a chain-saturated glycolipid with a lactose headgroup (N-palmitoyl-lactosylceramide, LacCer-sat, Figure 2A), a chainsaturated glycolipid with a trihexose headgroup (Trihexo-sat, Figure S4), and a glucosylated sterol ( $\beta$-D-glucosyl sitosterol, Glu-sitosterol, Figure 2F) are studied. Grazing-incidence X-ray diffraction (GIXD) reveals the structural ordering of the monolayers down to an Angstrom level. Complementary totalreflection X-ray fluorescence (TRXF) measurements enable quantification of the monolayers' preferential interactions with ions. The aqueous subphases contain $c_{0}=1 \mathrm{mM} \mathrm{KI}$, CsBr, or $\mathrm{CaBr}_{2}$. These salts have been chosen because of their pronounced effects on wood swelling ${ }^{6}$ and their good detectability by TRXF.

The monolayer of MGDG-sat (Figure 1A) is defined by three diffraction peaks in the wide-angle region (at high $Q_{x y}$ ) above the horizon $\left(Q_{z}>0\right)$, characterizing an oblique lattice structure of tilted chains $\left(t=31^{\circ}\right.$, Figure $1 \mathrm{~B}-\mathrm{D}$ and Supporting Information tables), as often encountered for optically active compounds. ${ }^{10,11}$ Neither lateral compression to higher surface pressure $\Pi$ nor the type of subphase significantly change the lattice structure (Figures 3 and S6). The small cross-sectional chain area of only $A_{0}=18.6 \AA^{2}$ indicates very tight packing with no rotational freedom. The in-plane

Received: December 28, 2018

Accepted: March 25, 2019

Published: March 25, 2019 

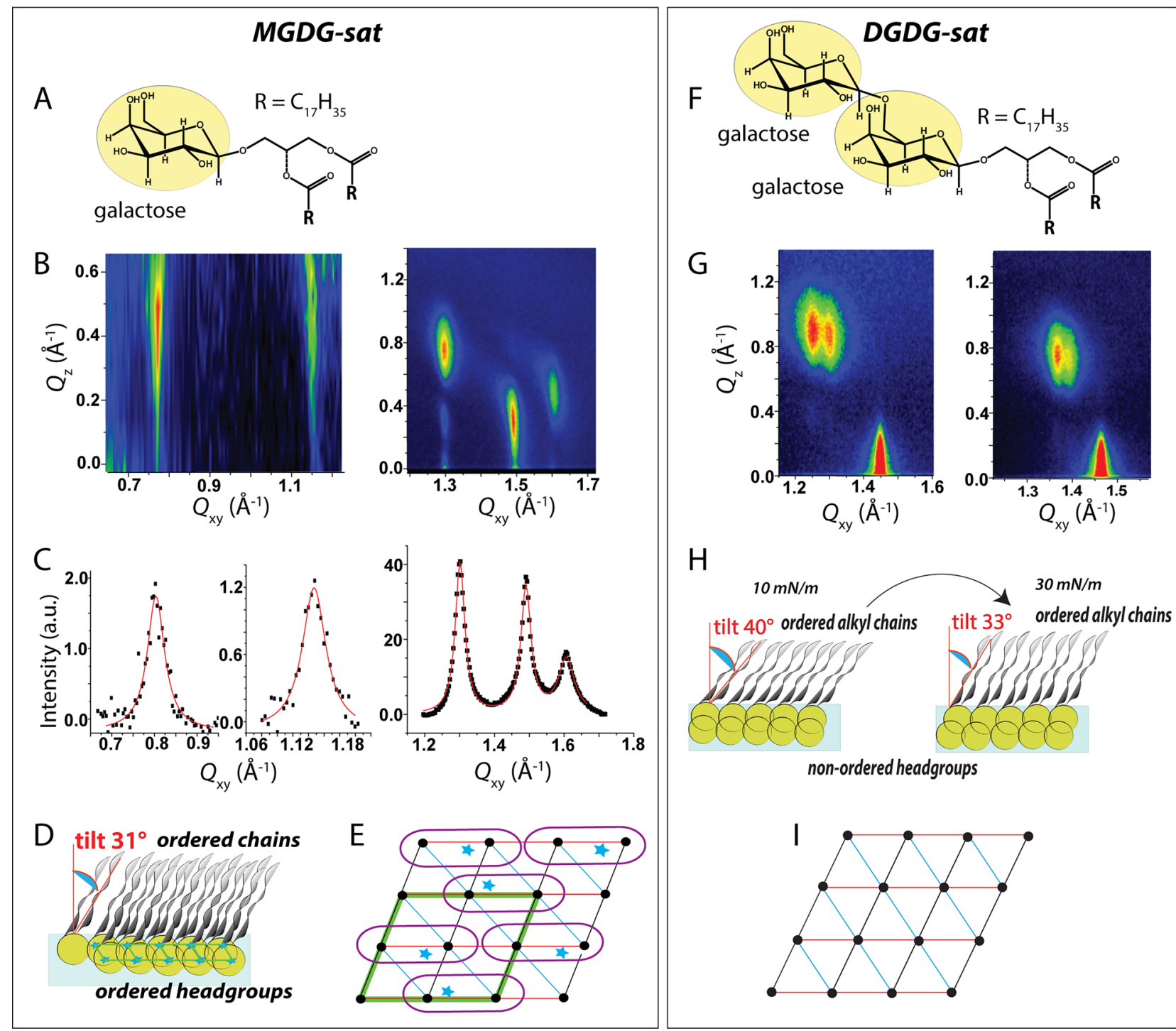

Figure 1. (A,F) Chemical structures of MGDG-sat and DGDG-sat. (B,G) GIXD contour plots displaying the scattered intensity versus the in-plane and the out-of-plane components of the scattering vector, $Q_{x y}$ and $Q_{y}$, respectively, obtained for $M G D G$-sat $(\Pi=30 \mathrm{mN} / \mathrm{m})$ and $D G D G$-sat $(\Pi=$ 10 and $30 \mathrm{mN} / \mathrm{m}$ ) monolayers on $1 \mathrm{mM} \mathrm{CsBr}$. (C) Bragg peaks obtained for MGDG-sat. (D,H) Schematic side-view of MGDG-sat and DGDG-sat monolayers, respectively, on the water surface. (E) Schematic top-view representation of the lattice formed by MGDG-sat. The positions of chains and headgroups are indicated with black dots and blue stars, respectively. Red, black, and blue triangles indicate the repeating unit cell of the alkyl chains. The unit cell of the molecule lattice is indicated with a green parallelogram. Violet line: delimitation of the molecules. (I) Schematic topview representation of the lattice formed by the alkyl chains of DGDG-sat.

molecular area, $A=2 A_{0} / \cos (t)=43.4 \AA^{2}$, allows the galactose moiety of $M G D G$-sat to orient parallel to the interface (the inplane area of the sugar headgroup is reported to be 35.4 $\left.\AA^{2}\right),{ }^{12,13}$ in agreement with previously reported data. ${ }^{14}$ Interestingly, two additional Bragg peaks are seen in the midangle region, i.e., at lower $Q_{x y}$ (Figure 1B,C). These peaks indicate an ordering of weakly hydrated galactose moieties, ${ }^{15}$ in good agreement with previous SAXS and WAXS data. ${ }^{16,17} \mathrm{~A}$ supercell indicating the ordering of entire molecules is identified (Figure 1E and Table S3). It is induced by strong intermolecular hydrogen bonds between the sugar headgroups, similar to the previously reported monolayer structure of a GPI fragment, ${ }^{18-20}$ although the existence of a superlattice in principle does not require crystalline ordering of the entire headgroups. This supercell (green parallelogram), reminiscent of subgel phases in bulk, ${ }^{21-23}$ is commensurate with the hydrocarbon chain lattice $\left(a^{\prime}=2 \times a_{\text {chains }}, b^{\prime}=2 \times b_{\text {chains }}, \gamma=\right.$ $110.1^{\circ}$ ) and, with an area of $86.8 \AA^{2}$, contains two MGDG-sat molecules. The rigid network of hydrogen bonds between galactose headgroups dictates the packing order of the chains (no change upon lateral compression). The full width at halfmaximum (fwhm) of the Bragg rods (SI) agrees well with the length of an extended C18 alkyl chain in all-trans conformation, confirming that the interfacial layer is a monolayer at all investigated surface pressures. ${ }^{24,25}$

MGDG-unsat does not form ordered monolayers. Gel phases in 3D systems have been found only at extremely low temperatures $\left(-30{ }^{\circ} \mathrm{C}\right) .^{26}$ Obviously, the highly ordered structure in MGDG-sat monolayers is a synergetic result of concomitant headgroup and chain interactions.

$D G D G$-sat does not exhibit diffraction peaks in the mid-towide-angle region, indicating the absence of headgroup order. Only the three diffraction peaks defining the alkyl chain lattice are observed in the wide-angle region (Figure 1G). The bulky 

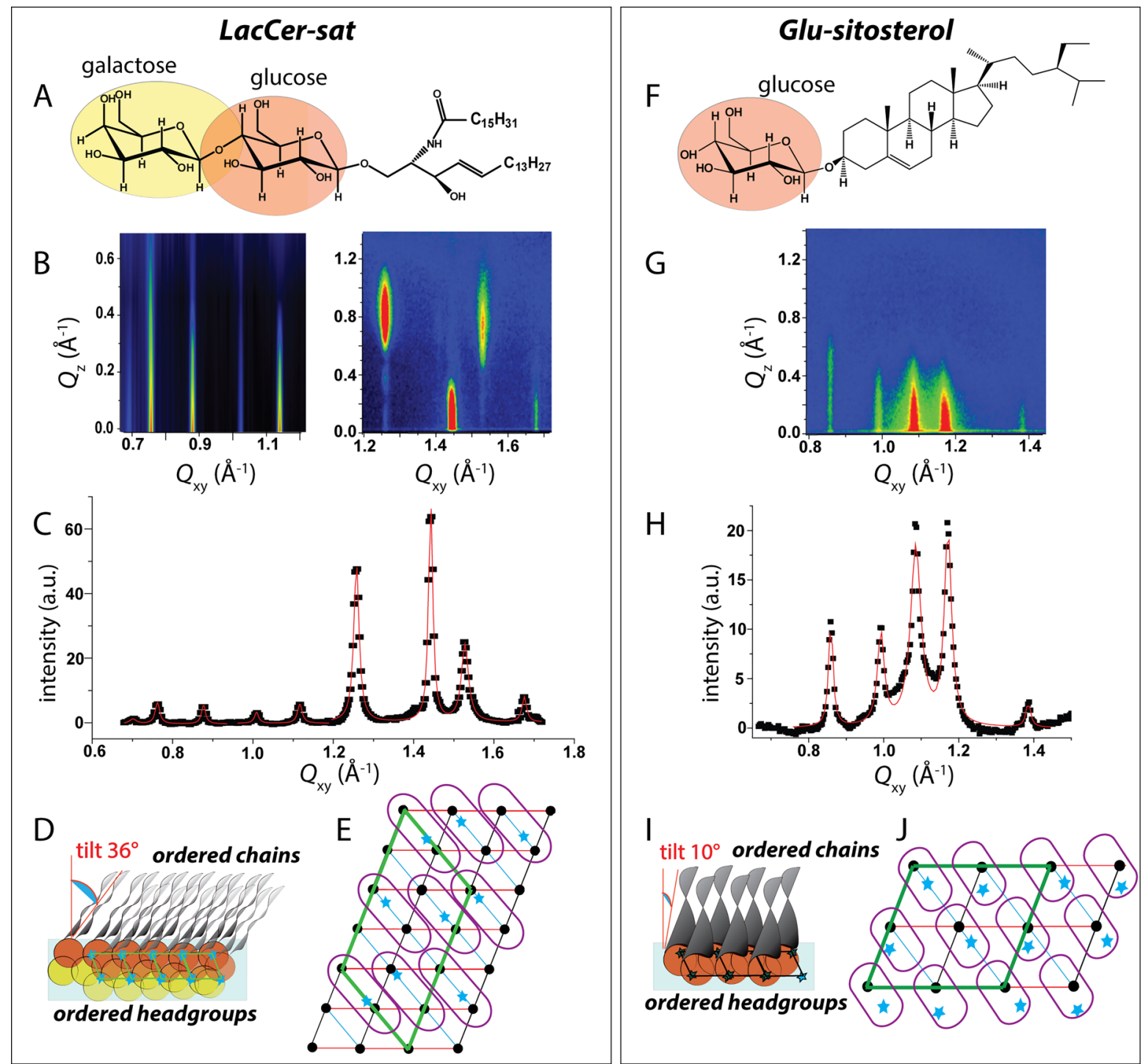

Figure 2. (A,F) Chemical structures of LacCer-sat and Glu-sitosterol. (B,G) GIXD contour plots obtained for LacCer-sat (10 mN/m, $1 \mathrm{mM}$ CsBr) and Glu-sitosterol $(30 \mathrm{mN} / \mathrm{m}, 1 \mathrm{mM} \mathrm{KI})$ monolayers, respectively. $(\mathrm{C}, \mathrm{H})$ Bragg peaks obtained for LacCer-sat $(10 \mathrm{mN} / \mathrm{m})$ and Glu-sitosterol (30 $\mathrm{mN} / \mathrm{m}$ ) monolayers, respectively. (D,I) Schematic side-view of LacCer-sat and Glu-sitosterol monolayers, respectively. (E,J) Schematic top-view representation of the lattices formed by LacCer-sat and Glu-sitosterol, respectively. The positions of chains and headgroups are indicated with black dots and blue stars, respectively. Red, black, and blue triangles indicate the repeating unit cell of the alkyl chains. The repeating unit cell of the headgroup/chain superlattice is indicated with a green parallelogram. Violet line: delimitation of the molecules.

digalactose moiety of DGDG-sat seems to disturb the packing of the headgroups, presumably due to a higher hydration degree, and offers higher flexibility to the molecules (noticeable decrease of the tilt angle during compression). The larger area requirement mismatch between the headgroup and chains leads to a higher tilt angle of the chains (Figure $1 \mathrm{H}$ and Tables S7 and S9). The lack of strong H-bonded and structured headgroups of DGDG-sat is in agreement with previous reports showing that a disaccharide sugar headgroup in glycerol-based glycolipids dramatically lowers the phase transition temperature compared to the corresponding molecule with a monosaccharide headgroup. ${ }^{15,27,28}$ Because the DGDG-sat in-plane molecular area is only slightly larger than that of MGDG-sat, the two sugar moieties cannot arrange parallel to the interface but more likely adopt a perpendicular or tilted arrangement. This is in agreement with previous infrared reflection-absorption spectroscopy (IRRAS) studies reporting a tilt angle of the digalactosyl headgroups of $\approx 40^{\circ}$ (ref 14). GIXD data recorded for monolayers of DGDG-unsat display no diffraction peaks at all, indicating disordered chains and headgroups.

To tackle the problem of sugar specificity for headgroup interactions, LacCer-sat is investigated. As DGDG-sat, LacCersat features two sugar moieties but of a different nature (galactose and glucose units) and forms condensed monolayers at room temperature on the surface of $1 \mathrm{mM}$ aqueous salt solutions. Yet, the GIXD data revealed a much more complex diffraction pattern with a multitude of peaks (Figure $2 \mathrm{~B}, \mathrm{C})$. Contrary to $M G D G$ and $D G D G$, a stronger influence of the subphase (Figure S6) and the formation of different polymorphs are observed. On a $1 \mathrm{mM}$ CsBr subphase, the three intense diffraction peaks in the wide-angle region can be attributed to the alkyl chain order, while the five additional weaker peaks in the mid-to-wide-angle region indicate headgroup ordering. The existence of a headgroup order in monolayers $^{29}$ and in bulk crystals ${ }^{30}$ of synthetic glycolipids 


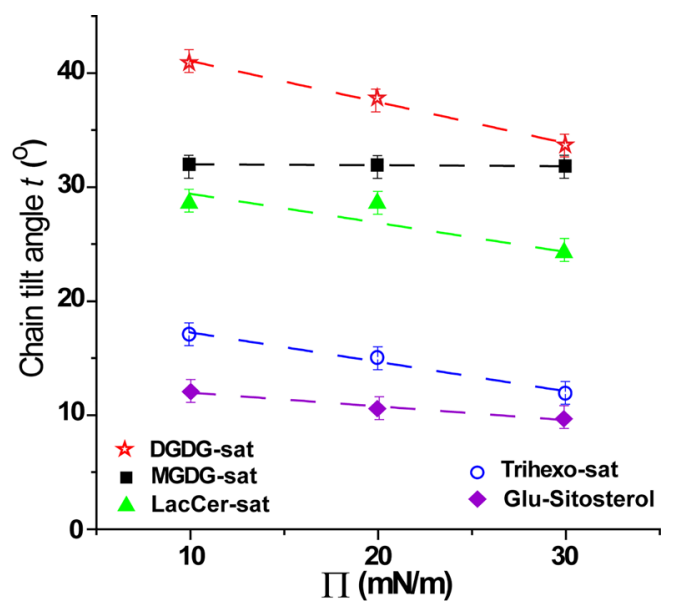

Figure 3. Variation of the tilt angle with the lateral pressure of the structured glycolipid monolayers on $1 \mathrm{mM} \mathrm{CaBr}_{2}$.

bearing lactose units has already been reported. Thus, it seems that lactose headgroups are more prone to be engaged in intermolecular interactions ${ }^{31}$ and layer structuring than the digalactosyl headgroups. The supercell (Figure 2E, green parallelogram) defines an area corresponding to four LacCersat molecules (Tables S10-S12). The H-bonding for LacCersat seems to be more complex due to the possible additional contribution of amide-sugar interactions. ${ }^{32,33}$ Different polymorphs (Tables S13-S16 and Figure S3) have been observed but will not be discussed here in detail. Literature agrees well with the tendency of LacCer-sat in forming different polymorphs in 3D systems. ${ }^{34,35}$ The Debye-Scherrer rings, seen only in the diffraction pattern at high surface pressures ( $\Pi$
$=30 \mathrm{mN} / \mathrm{m}$, SI Figure S3), indicate the formation of $3 \mathrm{D}$ crystals coexisting with a monolayer at the air/water interface. Such strong lactose-lactose interactions could be responsible for the formation of LacCer-enriched microdomains in biological systems (cell surface plasma membranes of mouse neutrophils, microdomains presenting a high specificity for antibodies). ${ }^{36}$

Monolayers of Trihexo-sat, which features the bulkiest headgroup, are found to be characterized by ordered alkyl chains and nonordered headgroups. Interestingly, this compound exhibits the lowest alkyl chain tilt, $t \approx 20^{\circ}$ at 10 $\mathrm{mN} / \mathrm{m}$, which further decreases to $t \lesssim 15^{\circ}$ at $30 \mathrm{mN} / \mathrm{m}$, depending on the subphase (Tables S17-22 and Figure S6).

The role played by the glucose unit in headgroup interactions is investigated in Glu-sitosterol monolayers (monoglucose-based glycolipids with saturated alkyl chains are not commercially available). The GIXD data reveal a monolayer structure defined by five Bragg peaks (Figure $2 \mathrm{G}, \mathrm{H})$. Three of them describe the order of the cholesterol moieties, and the additional Bragg peaks indicate headgroup order. The observed superstructure with an area of $156.3 \AA^{2}$ corresponds to four Glu-sitosterol molecules (Table S25). The cross-sectional area per molecule of $38.4 \AA^{2}$ is in good agreement with values of $37.7 \AA^{2}$ obtained for pure cholesterol. $^{25}$ This in-plane area allows the sugar headgroup to adopt a parallel orientation to the interface. Complex GIXD patterns have been often described in the literature and even for Langmuir monolayers of cholesterol, attributed to the formation of multilayers. ${ }^{25}$ In the present case, we refute such a scenario based on stable compression isotherms (Figure S5) and on the fwhm value of the Bragg rods (Table S23) corresponding to a monolayer. Similar to LacCer-sat, the Glu-
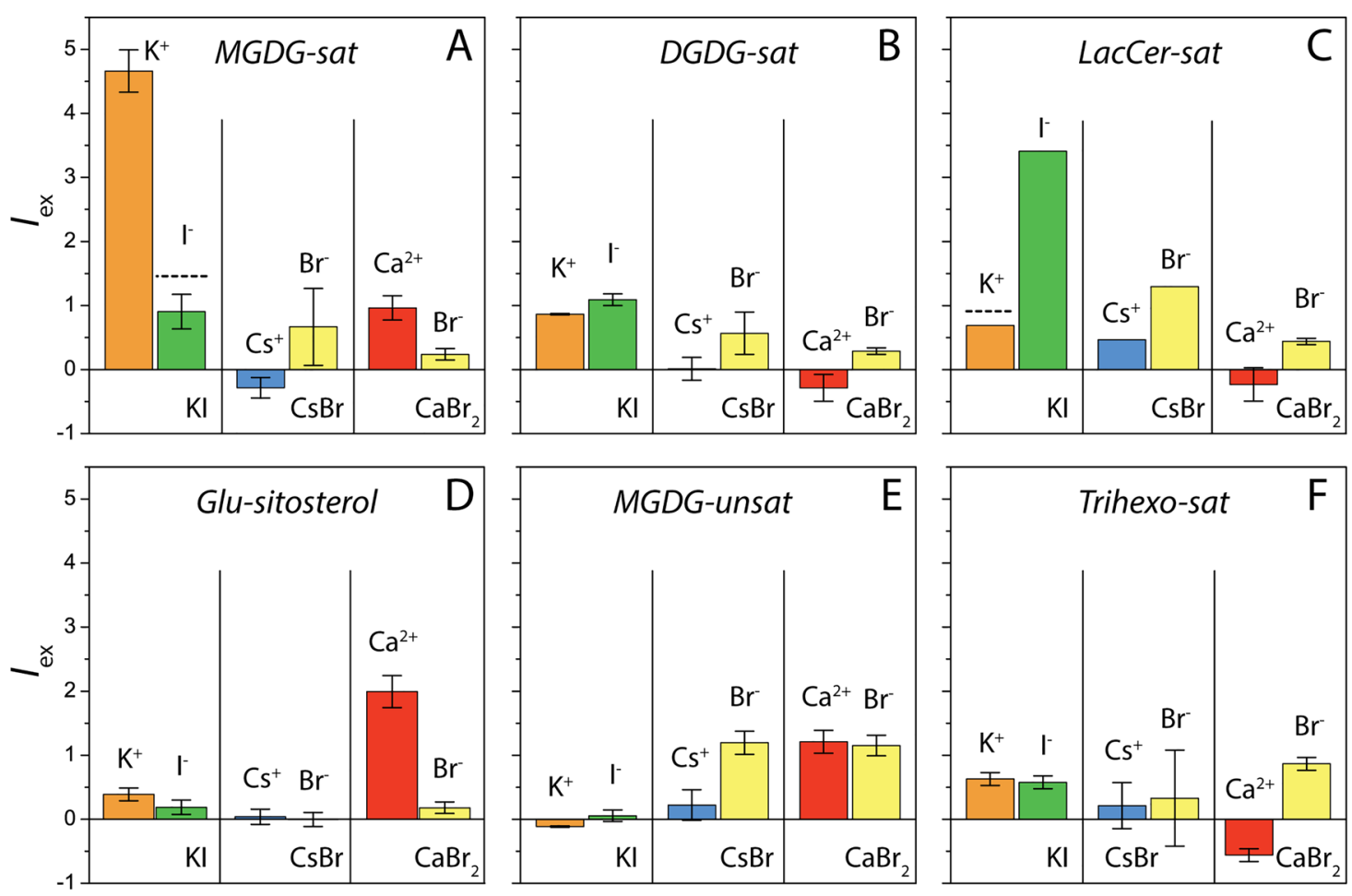

Figure 4. Relative excess fluorescence intensities of $\mathrm{K}^{+}, \mathrm{I}^{-}, \mathrm{Cs}^{+}, \mathrm{Br}^{-}$, and $\mathrm{Ca}^{2+}$ near various uncharged glycolipid monolayers on aqueous subphases containing $1 \mathrm{mM} \mathrm{KI}, \mathrm{CsBr}$, or $\mathrm{CaBr}_{2}$. Data are averages over up to three measurements at $\Pi=10,20$, and $30 \mathrm{mN} / \mathrm{m}$. Error bars represent one standard deviation. They are absent when only one data point was available. Horizontal dashed lines in panels (A) and (C) indicate the intensity level expected from a generic charge-neutralization effect (see the main text). 
sitosterol monolayers exhibit stronger response to the subphase nature (Figure S6). Polymorphs of different thermodynamic stability, possibly induced by headgroup hydration/dehydration, ${ }^{37}$ are observed but again will not be discussed here in detail.

Overall, the investigated electrically neutral glycolipid monolayers can be divided into three classes: (i) highly structured monolayers, characterized by ordered headgroups and ordered alkyl chains (MGDG-sat, LacCer-sat, and Glusitosterol), (ii) structured monolayers with ordered alkyl chains but disordered headgroups (DGDG-sat, Trihexo-sat), and (iii) nonstructured monolayers with no headgroup and no chain order (MGDG-unsat and DGDG-unsat). Classes (i) and (ii) are distinguishable also by the variation of the tilt angle of the alkyl chains with the surface pressure (Figure 3 ). The highly structured monolayers in tendency exhibit less variation of the tilt angle due to the rigid headgroup lattice. Those are considered to have a low degree of hydration and to strongly interact, forming a network of hydrogen bonds. ${ }^{37} \mathrm{~A}$ more pronounced gradual decrease of the chain tilt angle with increasing lateral pressure is recorded for the structured monolayers characterized by only ordered alkyl chains. This behavior points to a higher flexibility of the monolayer and of the molecular interactions associated with a higher degree of headgroup hydration. ${ }^{38}$

TRXF experiments were carried out in order to quantify preferential interactions of ions with the glycolipid monolayers in terms of interfacial ion excesses per unit area. The method is highly sensitive to interfacial excesses because the X-ray beam is totally reflected and only illuminates the immediate vicinity of the interface with an evanescent wave. ${ }^{39-42}$ The excess of each ion type is then deduced from the intensity of its elementcharacteristic X-ray fluorescence. Importantly, in the presence of the $1 \mathrm{mM}$ salt used in the TRXF experiments, the Debye length $\left(\kappa^{-1} \approx 10 \mathrm{~nm}\right.$ for $\mathrm{KI}$ and $\mathrm{CsBr}, \kappa^{-1}=6 \mathrm{~nm}$ for $\left.\mathrm{CaBr}_{2}\right)$ is comparable to the intensity decay length of the evanescence wave $(\Lambda \approx 7 \mathrm{~nm})$ for the used combination of incident angle $\left(\theta_{\mathrm{i}}=0.11\right.$ or $\left.0.07^{\circ}\right)$ and beam energy $(E=8.0$ or $15.0 \mathrm{keV})$. The fluorescence intensity of an ion species preferentially interacting with the surface is therefore higher than that of the corresponding counterion species, which approximately obeys a Gouy-Chapman distribution to achieve charge neutrality on the length scale of $\kappa^{-1}$ (ref 43) but does not reach a 1:1 ion stoichiometry within the illuminated volume. As a consequence, the measurements allow identification of the preferentially interacting ion species for each salt type.

Figure 4 shows the relative excess fluorescence intensities, $I_{\mathrm{ex}}$ of $\mathrm{K}^{+}, \mathrm{I}^{-}, \mathrm{Cs}^{+}, \mathrm{Br}^{-}$, and $\mathrm{Ca}^{2+}$ near monolayers of $M G D G$ sat, DGDG-sat, LacCer-sat, Glu-sitosterol, MGDG-unsat, and Trihexo-sat on aqueous subphases containing $1 \mathrm{mM} \mathrm{KI}, \mathrm{CsBr}$, or $\mathrm{CaBr}_{2}$. Data are averages over up to three measurements at $\Pi=10,20$, and $30 \mathrm{mN} / \mathrm{m} . I_{\mathrm{ex}}=\left(I-I_{0}\right) / I_{0}$ is the relative change in the measured intensity $I$ with respect to the intensity $I_{0}$ expected in the absence of any preferential interactions, i.e., assuming bulk-like ion concentration up to the monolayer surface. $I_{0}$, in turn, is obtained by measuring the intensity from the bare aqueous subphase, $I_{\text {bare, }}$ and taking into account the reduction of the illuminated aqueous volume in the presence of the monolayer in the form of a prefactor $f, I_{0}=f \cdot I_{\text {bare }}$. This prefactor is given by the electron density profile of the monolayer and robust with respect to minor uncertainties in the characteristics of these profiles (SI). The ion distributions at the bare air/water interface are approximated as constant, neglecting minor deviations ${ }^{39,44}$ affecting $I_{\text {bare }}$ only by few percent. $^{39}$ As shown in the SI, the employed methodology is consistent with an absolute intensity calibration using charged monolayers.

Positive values of $I_{\mathrm{ex}}$ in Figure 4 correspond to an accumulation of the respective ion species at the respective monolayer, and negative values correspond to a depletion. It is seen that significant ion accumulation occurs only for certain monolayer/salt combinations. Strong accumulation is observed for $\mathrm{K}^{+}$near monolayers of MGDG-sat, which implies preferential interactions of $\mathrm{K}^{+}$with the monolayer surface. The moderate excess of the counterion $\mathrm{I}^{-}$, on the other hand, must be interpreted as a secondary effect of charge neutralization. The horizontal dashed line in Figure 4A indicates the intensity level expected from this secondary effect within a Poisson-Boltzmann model described in the SI. The measured $\mathrm{I}^{-}$excess is somewhat below this estimate, suggesting even slightly unfavorable interactions of $\mathrm{I}^{-}$with the interface. The magnitude of the $\mathrm{K}^{+}$excess, as deduced from $I_{\mathrm{ex}}$ within the model, is $\Gamma_{\mathrm{K}} \approx 0.02 \mathrm{~nm}^{-2}$, corresponding to an area per adsorbed ion of $A_{\mathrm{K}}=1 / \Gamma_{\mathrm{K}} \approx 50 \mathrm{~nm}^{2}$ or 1 ion per approximately 100 lipids. This excess is roughly 2 orders of magnitude smaller than previously measured ion excesses compensating a certain number of charges per lipid in charged lipid monolayers. ${ }^{41,42}$ Nonetheless, the preferential interaction of $\mathrm{K}^{+}$with the charge-neutral monolayer is significant. At this point, it should be noted that neither charged impurities nor monolayer ionization by X-ray irradiation can be the cause for the observed accumulation because other cations including the divalent $\mathrm{Ca}^{2+}$ ions do not accumulate significantly at the same surface under the same conditions (Figure 4A). The same reasoning holds for other monolayer/salt combinations. A similar extent of ion accumulation is found for $\mathrm{KI}$ near monolayers of LacCer-sat (Figure 4C). Interestingly, the situation is reversed: $\mathrm{I}^{-}$instead of $\mathrm{K}^{+}$ions exhibit preferential interactions with this interface, with an ion excess of $\Gamma_{I} \approx$ $0.014 \mathrm{~nm}^{-2}$. The horizontal dashed line again indicates the intensity level expected from a generic charge-neutralization effect. Pronounced ion accumulation is also found for $\mathrm{Ca}^{2+}$ near Glu-sitosterol monolayers (Figure 4D), with $\Gamma_{\mathrm{Ca}} \approx 0.012$ $\mathrm{nm}^{-2}$. The depth of the ion-adsorbing potential, $\Delta G$, can be estimated from $\Gamma$ via Boltzmann inversion, $\Delta G=-k_{\mathrm{B}} T \ln (\Gamma /$ $\left.c_{0} d\right)$, where $k_{\mathrm{B}}$ is the Boltzmann constant and $d$ the width of the ion-adsorbing region. For a reasonable $d$ range $(2 \AA<d<7$ $\AA$ ), we obtain $\Delta G=-11.2 \pm 2 \mathrm{~kJ} / \mathrm{mol}\left(\mathrm{K}^{+}\right.$at $M G D G$-sat $), \Delta G$ $=-10.3 \pm 2 \mathrm{~kJ} / \mathrm{mol}\left(\mathrm{I}^{-}\right.$at LacCer-sat $)$, and $\Delta G=-9.7 \pm 2 \mathrm{~kJ} /$ $\mathrm{mol}\left(\mathrm{Ca}^{2+}\right.$ at Glu-sitosterol). These numbers roughly correspond to $1 / 3$ of the free energy per hydrogen bond in water. ${ }^{45}$

All monolayers exhibiting pronounced preferential interactions with at least one ion species belong to monolayer class (i) featuring headgroup order. On the other hand, monolayers of classes (ii) or (ii), without headgroup order, do not seem to exhibit any clear trends. This notion suggests that the defined structural motifs displayed by headgroup-ordered surfaces are responsible for the pronounced ion selectivity. It further provides a possible explanation for the observation that different headgroup chemistries, which lead to different structural motifs, exhibit selectivity for different ions. The selectivity likely arises due to a match between the hydrogen bond configurations inside of the defined saccharide "pockets" and those of the ions' hydration shells, which are known to be species-dependent. With that, the observed phenomenon appears to be related to the remarkable ion selectivity of 
crown ethers owing to their characteristic polyether cavity. ${ }^{46}$ Indeed, crown ethers such as dibenzo-18-crown-6 (18C6), an 18 -atom heterocycle containing 6 oxygen atoms, selectively capture $\mathrm{K}^{+}$, while other polyether rings like 15-crown-5 (15C5) or 21-crown-7 (21C7) selectively capture $\mathrm{Na}^{+}$ $\left(\mathrm{Ca}^{2+}\right)$ and $\mathrm{Cs}^{+}$ions, respectively. ${ }^{47,48}$ Our results demonstrate impressively that uncharged hydrophilic surfaces in general and saccharide surfaces in particular can selectively attract ions, the species being dependent on the structural motifs displayed by the surfaces. At first glance, the determined ion excesses appear to be weak. Note, however, that the bulk ion concentrations in the present study were chosen to be very low. The excess increases with the bulk concentration (albeit underproportionally) and therefore can be expected to reach considerable levels at biologically or technologically relevant concentrations. Selectivity to only one ion species in a salt solution inevitably leads to a charge separation that, in turn, results in electrostatic repulsion between two such surfaces. ${ }^{6,49}$ This notion provides a route to a better understanding of the ion-specificity in the swelling of wood. ${ }^{6}$ In a biological context, the effective surface charge induced by preferential interactions of ions with headgroup-ordered glycolipid microdomains ("lipid rafts") ${ }^{50}$ is suited to attract proteins and to accelerate their binding. Moreover, such preferential ion interactions lead to additional coupling between the lateral and perpendicular equations of state of multilamellar membrane systems. ${ }^{51}$ Our results motivate further systematic investigations with the aim to identify correlations between the structural features of the crystalline saccharide surfaces and the preferentially adsorbing ion species. In the longer term, atomistic molecular dynamics simulations appear to be suited to shed additional light on the underlying physical mechanisms.

\section{ASSOCIATED CONTENT}

\section{S Supporting Information}

The Supporting Information is available free of charge on the ACS Publications website at DOI: 10.1021/acs.jpclett.8b03865.

Additional information on methods and results, including fluorescence studies, additional calculations, modeling of the electron density profiles, prediction of ion excesses, GIXD results, chemical structure representations, Langmuir compression isotherms, and peak indexing, (PDF)

\section{AUTHOR INFORMATION}

\section{Corresponding Author}

*E-mail: schneck@mpikg.mpg.de. Phone: +49-331567-9404. Fax: +49-331567-9402.

\section{ORCID}

Victoria M. Latza: 0000-0002-1763-2712

Philippe Fontaine: 0000-0003-3394-6508

Emanuel Schneck: 0000-0001-9769-2194

Notes

The authors declare no competing financial interest.

\section{ACKNOWLEDGMENTS}

We thank Deutsches Elektronen-Synchrotron (DESY) and Synchrotron SOLEIL for beamtime allocation and Pierre Bauduin for insightful comments. Financial support by the Max Planck Society and by the German Research Foundation
(DFG) via an Emmy-Noether grant (SCHN 1396/1) is gratefully acknowledged.

\section{REFERENCES}

(1) Gabius, H. J.; Roth, J. An introduction to the sugar code. Histochem. Cell Biol. 2017, 147 (2), 111.

(2) Bucior, I.; Burger, M. M. Carbohydrate-carbohydrate interactions in cell recognition. Curr. Opin. Struct. Biol. 2004, 14 (5), 631637.

(3) Lis, H.; Sharon, N. Lectins: carbohydrate-specific proteins that mediate cellular recognition. Chem. Rev. 1998, 98 (2), 637-674.

(4) Nodet, G.; Poggi, L.; Abergel, D.; Gourmala, C.; Dong, D.; Zhang, Y.; Mallet, J.-M.; Bodenhausen, G. Weak calcium-mediated interactions between Lewis X-related trisaccharides studied by NMR measurements of residual dipolar couplings. J. Am. Chem. Soc. 2007, 129 (29), 9080-9085.

(5) Geyer, A.; Gege, C.; Schmidt, R. R. Calcium-Dependent Carbohydrate-Carbohydrate Recognition between LewisX Blood Group Antigens. Angew. Chem., Int. Ed. 2000, 39 (18), 3245-3249.

(6) Barbetta, A.; Fratzl, P.; Zemb, T.; Bertinetti, L. Impregnation and Swelling of Wood with Salts: Ion Specific Kinetics and Thermodynamics Effects. Adv. Mater. Interfaces 2017, 4 (1), 1600437.

(7) Pantelic, I.; Cuckovic, B. Alkyl Polyglucosides: From NaturalOrigin Surfactants to Prospective Delivery Systems; Woodhead Publ Ltd., Cambridge, England, 2014; Vol. 70.

(8) Datta, S.; Bhattacharya, S. Multifarious facets of sugar-derived molecular gels: molecular features, mechanisms of self-assembly and emerging applications. Chem. Soc. Rev. 2015, 44, 5596.

(9) Block, M. A.; Dorne, A.-J.; Joyard, J.; Douce, R. Preparation and characterization of membrane fractions enriched in outer and inner envelope membranes from spinach chloroplasts. II. biochemical characterization. J. Biol. Chem. 1983, 258, 13281-13286.

(10) Brezesinski, G.; Dietrich, A.; Struth, B.; Boehm, C.; Bouwman, W. G.; Kjaer, K.; Moehwald, H. Influence of ether linkages on the structure of double-chain phospholipid monolayers. Chem. Phys. Lipids 1995, 76, 145-157.

(11) Scalas, E.; Brezesinski, G.; Kaganer, V. M.; Moehwald, H. Effect of chiral interactions on the structure of Langmuir monolayers. Phys. Rev. E: Stat. Phys., Plasmas, Fluids, Relat. Interdiscip. Top. 1998, 58 (2), 2172.

(12) Moews, P. C.; Knox, J. R. D-glucopyranoside: a polar bilayer with a hydrocarbon subcell. J. Am. Chem. Soc. 1976, 98, 6628.

(13) Dorset, D. L.; Rosenbusch, J. P. Solid State Properties of Anomeric I-O-n-Octyl-D-Glucopyranosides. Chem. Phys. Lipids 1981, 29, 299-307.

(14) Bottier, C.; Géan, J.; Artzner, F.; Desbat, B.; Pézolet, M.; Renault, A.; Marion, D.; Vié, V. Galactosyl headgroup interactions control the molecular packing of wheat lipids in Langmuir films and in hydrated liquid-crystalline mesophases. Biochim. Biophys. Acta, Biomembr. 2007, 1768, 1526-1540.

(15) Sen, A.; Mannock, D. A.; Collins, D. J.; Quinn, P. J.; Williams, W. P. Thermotropic phase properties and structure of 1,2distearoylgalactosylglycerols in aqueous systems. Proc. R. Soc. London B 1983, 218, 349-364.

(16) Lis, L. J.; Quinn, P. J. A time-resolved synchrotron X-ray study of a crystalline phase bilayer transition and packing in a saturated monogalactosyldiacylglycerol-water system. Biochim. Biophys. Acta, Biomembr. 1986, 862, 81-86.

(17) Quinn, P. J.; Lis, L. J. A Characterization of the Phase Transitions in Saturated Monogalactosyldiacylglycerol-Water Systems Using Real-Time X-Ray Diffraction Methods. Mol. Cryst. Liq. Cryst. 1989, 167 (1), 109-121.

(18) Stefaniu, C.; Vilotijevic, I.; Brezesinski, G.; Seeberger, P. H.; Varon Silva, D. A comparative structural study in monolayers of GPI fragments and their binary mixtures. Phys. Chem. Chem. Phys. 2014, 16,9259 .

(19) Stefaniu, C.; Vilotijevic, I.; Santer, M.; Brezesinski, G.; Seeberger, P. H.; Varon Silva, D. Versatility of a Glycosylphospha- 
tidylinositol Fragment in Forming Highly Ordered Polymorphs. Langmuir 2014, 30, 5185-5192.

(20) Stefaniu, C.; Vilotijevic, I.; Santer, M.; Varon Silva, D.; Brezesinski, G.; Seeberger, P. H. Subgel Phase Structure in Monolayers of Glycosylphosphatidylinositol Glycolipids. Angew. Chem., Int. Ed. 2012, 51, 12874-12878.

(21) Katsaras, J.; Raghunathan, V. A.; Dufourc, E. J.; Dufourcq, J. Biochemistry 1995, 34, 4684.

(22) Marsh, D. Chem. Phys. Lipids 2012, 165, 59.

(23) Albon, N. J. Chem. Phys. 1983, 79, 469.

(24) Kuzmenko, I.; Kindermann, M.; Kjaer, K.; Howes, P. B.; AlsNielsen, J.; Granek, R.; von Kiedrowski, G.; Leiserowitz, L.; Lahav, M. Crystalline films of interdigitated structures formed via amidiniumcarboxylate interactions at the air-water interface. J. Am. Chem. Soc. 2001, 123, 3771.

(25) Rapaport, H.; Kuzmenko, I.; Lafont, S.; Kjaer, K.; Howes, P. B.; Als-Nielsen, J.; Lahav, M.; Leiserowitz, L. Cholesterol monohydrate nucleation in ultrathin films on water. Biophys. J. 2001, 81, 2729.

(26) Sanderson, P. W.; Williams, W. P. Low-temperature phase behaviour of the major plant leaf lipid monogalactosyldiacylglycerol. Biochim. Biophys. Acta, Biomembr. 1992, 1107, 77-85.

(27) Sen, A.; Hui, S.-W.; Mannock, D. A.; Lewis, R. N. A. H.; McElhaney, R. N. Physical properties of glycosyldiacylglycerols. 2. Xray diffraction studies of a homologous series of 1,2-Di-O-acyl-3-O-(aD-glucopyranosyl)-sn-glycerols. Biochemistry 1990, 29, 7799-7804.

(28) Sen, A.; Williams, W. P.; Quinn, P. J. The structure and thermotropic properties of pure 1,2-diacylgalactosylglycerols in aqueous systems. Biochim. Biophys. Acta, Lipids Lipid Metab. 1981, 663, 380-389.

(29) Tanaka, M.; Schneider, M. F.; Brezesinski, G. In-Plane Structures of Synthetic Oligolactose Lipid Monolayers-Impact of Saccharide Chain Length. ChemPhysChem 2003, 4, 1316-1322.

(30) Schneider, M. F.; Zantl, R.; Gege, C.; Schmidt, R. R.; Rappolt, M.; Tanaka, M. Hydrophilic/Hydrophobic Balance Determines Morphology of Glycolipids with Oligolactose Headgroups. Biophys. J. 2003, 84, 306-313.

(31) Yu, Z.; Calvert, T. L.; Leckband, D. Molecular forces between membranes displaying neutral glycosphingolipids: evidence for carbohydrate attraction. Biochemistry 1998, 37, 1540-1550.

(32) Pascher, I. Molecular arrangements in sphingolipids conformation and hydrogen-bonding of ceramide and their implication on membrane stability and permeability. Biochim. Biophys. Acta, Biomembr. 1976, 455, 433-451.

(33) Ruocco, M. J.; Atkinson, D.; Small, D. M.; Skarjune, R. P.; Oldfield, E.; Shipley, G. G. X-ray Diffraction and Calorimetric Study of Anhydrous and Hydrated N-(Palmitoylgalactosylsphingosine Cerebroside). Biochemistry 1981, 20, 5957-5966.

(34) Li, X.-M.; Momsen, M.-M.; Brockman, H. L.; Brown, R. E. Lactosylceramide: Effect of Acyl Chain Structure on Phase Behavior and Molecular Packing. Biophys. J. 2002, 83, 1535-1546.

(35) Saxena, K.; Zimmermann, P.; Schmidt, R. R.; Shipley, G. G. Bilayer Properties of Totally Synthetic C16:0-Lactosyl-Ceramide. Biophys. J. 2000, 78, 306-312.

(36) Iwabuchi, K.; Masuda, H.; Kaga, N.; Nakayama, H.; Matsumoto, R.; Iwahara, C.; Yoshizaki, F.; Tamaki, Y.; Kobayashi, T.; Hayakawa, T.; Ishii, K.; Yanagida, M.; Ogawa, H.; Takamori, K. Properties and functions of lactosylceramide from mouse neutrophils. Glycobiology 2015, 25 (6), 655-668.

(37) Hinz, H.-J.; Kuttenreich, H.; Meyer, R.; Renner, M.; Fruend, R.; Koynova, R.; Boyanov, A. I.; Tenchov, B. G. Stereochemistry and Size of Sugar Head Groups Determine Structure and Phase Behavior of Glycolipid Membranes: Densitometric, Calorimetric, and X-ray Studies. Biochemistry 1991, 30, 5125-5138.

(38) Stefaniu, C.; Ries, A.; Gutowski, O.; Ruett, U.; Seeberger, P. H.; Werz, D. B.; Brezesinski, G. Impact of Structural Differences in Galactocerebrosides on the Behavior of 2D Monolayers. Langmuir 2016, 32, 2436-2444.

(39) Padmanabhan, V.; Daillant, J.; Belloni, L.; Mora, S.; Alba, M.; Konovalov, O. V. Specific Ion Adsorption and Short-Range
Interactions at the Air Aqueous Solution Interface. Phys. Rev. Lett. 2007, 99, 086105.

(40) Schneck, E.; Demé, B. Structural characterization of soft interfaces by standing-wave fluorescence with X-rays and neutrons. Curr. Opin. Colloid Interface Sci. 2015, 20 (4), 244-252.

(41) Schneck, E.; Schubert, T.; Konovalov, O. V.; Quinn, B. E.; Gutsmann, T.; Brandenburg, K.; Oliveira, R. G.; Pink, D. A.; Tanaka, M. Quantitative determination of ion distributions in bacterial lipopolysaccharide membranes by grazing-incidence X-ray fluorescence. Proc. Natl. Acad. Sci. U. S. A. 2010, 107 (20), 9147-9151.

(42) Shapovalov, V. L.; Ryskin, M. E.; Konovalov, O. V.; Hermelink, A.; Brezesinski, G. Elemental analysis within the electrical double layer using total reflection X-ray fluorescence technique. J. Phys. Chem. B 2007, 111, 3927-3934.

(43) Israelachvili, J. N. Intermolecular and Surface Forces; Academic Press, 2011.

(44) Horinek, D.; Herz, A.; Vrbka, L.; Sedlmeier, F.; Mamatkulov, S. I.; Netz, R. R. Specific ion adsorption at the air/water interface: the role of hydrophobic solvation. Chem. Phys. Lett. 2009, 479 (4-6), 173-83.

(45) Fersht, A. R.; Shi, J.-P.; Knill-Jones, J.; Lowe, D. M.; Wilkinson, A. J.; Blow, D. M.; Brick, P.; Carter, P.; Waye, M. M. Y.; Winter, G. Hydrogen bonding and biological specificity analysed by protein engineering. Nature 1985, 314 (6008), 235.

(46) Izatt, R. M.; Bradshaw, J. S.; Nielsen, S. A.; Lamb, J. D.; Christensen, J. J.; et al. Thermodynamic and kinetic data for cationmacrocycle interaction. Chem. Rev. 1985, 85, 271-339.

(47) Inokuchi, Y.; Boyarkin, O. V.; Kusaka, R.; Haino, T.; Ebata, T.; Rizzo, T. R. Ion Selectivity of Crown Ethers Investigated by UV and IR Spectroscopy in a Cold Ion Trap. J. Phys. Chem. A 2012, 116, 4057-4068.

(48) Pedersen, C. J. The Discovery of Crown Ethers. Science 1988, 241, 536-540.

(49) Schneck, E.; Demé, B.; Gege, C.; Tanaka, M. Membrane adhesion via homophilic saccharide-saccharide interactions investigated by neutron scattering. Biophys. J. 2011, 100 (9), 2151-2159.

(50) Kopitz, J. Lipid glycosylation: a primer for histochemists and cell biologists. Histochem. Cell Biol. 2017, 147, 175-198.

(51) Leontidis, E.; Aroti, A.; Belloni, L.; Dubois, M.; Zemb, T. Effects of monovalent anions of the Hofmeister series on DPPC lipid Bilayers part II: Modeling the perpendicular and lateral equation-ofstate. Biophys. J. 2007, 93 (5), 1591-1607. 